\title{
Why Do Document-Level Polarity Classifiers Fail?
}

\author{
Karen S. Martins \\ CS Department \\ UFMG
}

Belo Horizonte, MG, Brazil

karensmedcc.ufmg.br

\author{
Pedro O.S. Vaz-de-Melo \\ CS Department \\ UFMG
}

Belo Horizonte, MG, Brazil

olmo@dcc.ufmg.br

\author{
Rodrygo L. T. Santos \\ CS Department \\ UFMG
}

Belo Horizonte, MG, Brazil

rodrygo@dcc.ufmg.br

\begin{abstract}
Machine learning solutions are often criticized for the lack of explanation of their successes and failures. Understanding which instances are misclassified and why is essential to improve the learning process. This work helps to fill this gap by proposing a methodology to characterize, quantify and measure the impact of hard instances in the task of polarity classification of movie reviews. We characterize such instances into two categories: neutrality, where the text does not convey a clear polarity, and discrepancy, where the polarity of the text is the opposite of its true rating. We quantify the number of hard instances in polarity classification of movie reviews and provide empirical evidence about the need to pay attention to such problematic instances, as they are much harder to classify, for both machine and human classifiers. To the best of our knowledge, this is the first systematic analysis of the impact of hard instances in polarity detection from wellformed textual reviews.
\end{abstract}

\section{Introduction}

Document-level polarity classification is the task of classifying the polarity of a whole opinionated message (Pozzi et al., 2016). For instance, given a movie review, the system determines whether the review text expresses an overall positive, negative, or neutral opinion about the movie. Although polarity classification naturally suits to analyze consumer opinions about products and services (Gui et al., 2017), it is also well suited to various types of applications, such as to infer votes in elections (Goldberg et al., 2007), civilian sentiment during terrorism scenarios (Cheong and Lee, 2011), citizens' perception of government agencies (Arunachalam and Sarkar, 2013) and recommendation systems (Zhang, 2015).

Supervised machine learning is one of the most common and successful approaches for polarity classification, but even state-of-the-art methods fail to correctly classify a substantial portion of the instances, from $10 \%$ to $20 \%$, depending on the dataset (Ribeiro et al., 2016). The problem with this approach is that if the data is not representative and reliable, the model is unlikely to perform well. One source of unreliability is data noise, which can be categorized into class noise and attribute noise (Gupta and Gupta, 2019). Class noise occurs when the training data contains instances that are wrongly labeled. Attribute noise occurs when the training data contains one or more attributes with wrong, incomplete or missing values. In the case of textual data, such noise usually comes in the form of errors in language rules, such as typos, grammatical errors, improper punctuation, and abbreviations (Agarwal et al., 2007; Michel and Neubig, 2018; Lourentzou et al., 2019). Nevertheless, for both cases, the noise can be eliminated from the data by correcting the labels (for class noise) or the problematic text (for attribute noise).

A more problematic source of data unreliability in polarity classification tasks comes from well written text that, for some reason, does not convey its class clearly. Literature calls such instances hard instances, which are those that are intrinsically hard to correctly label or classify (Smith and Martinez, 2011; Beigman Klebanov and Beigman, 2014). Differently from noisy instances, hard instances cannot be corrected, so the only solution is to identify and remove them from the training data. Also, hard instances are not equivalent to outliers, as they do not differ significantly from other observations and may represent a significant portion of the data (Smith et al., 2014). For example, in a polarity classification task, a positive movie review that describes at least as many negative as positive points of the film can be a hard instance. To the best of our knowledge, no study exists that characterizes such instances and quantifies their impact on document-level polarity classification tasks.

Thus, we propose a methodology to characterize, 
quantify and measure the impact of hard instances in polarity classification tasks and demonstrate its usefulness in the task of movie review polarity classification. To this end, we collected 415, 867 positive and negative movie reviews from Metacritic. One advantage of Metacritic is that the meaning of ratings is clearly stated to the users when a review is being submitted: positive ratings range between $61 \%$ and $100 \%$, neutral range between $40 \%$ and $60 \%$, and negative between $0 \%$ and $39 \%$. Because of that, class noise and biases should be rare, that is, a user who liked (disliked) a movie will very unlikely give a negative (positive) rating to it. Thus, classification errors will mostly be due to hard instances, which we assign into two disjoint categories: neutral and discrepant. A neutral review does not have a clear polarity and a discrepant review has a human-perceived polarity that is different from its associated rating. This categorization is complete, i.e., every instance that, for a human, does not reveal its class clearly falls into one (and only one) of these two types of hard instances.

Neutral and discrepant reviews are characterized by a well-defined human classifier that uses human reasoning to infer the class of the example. When the class assigned by the human classifier is incorrect, we label the review as discrepant, i.e., the human-perceived polarity of the text is different from its associated rating. When the human classifier is not confident about its prediction, we label the review as neutral. We labeled 1,200 reviews and found 198 neutral and 64 discrepant reviews. We tested state-of-the-art machine classifiers on these reviews and results revealed that hard instances can significantly decrease their performances. In short, the main contributions are:

- A simple and reproducible methodology based on a well-defined human classifier to characterize and identify hard instances on polarity classification tasks (Section 3);

- A thorough analysis of the impact of hard instances in the task of movie review polarity classification (Section 5.2);

- Publicly available datasets of movie reviews describing the expected amounts of five classes of hard instances (Section 5.1).

As an additional contribution, we show how far are state-of-the-art machine classifiers from human performance in the task of movie review polarity classification.

\section{Related Work}

In supervised machine learning, class and attribute noise can increase learning complexity and, consequently, reduce classification accuracy (Zhu and $\mathrm{Wu}, 2004)$. Class noise is considered to be more harmful than attribute noise (Frenay and Verleysen, 2014), but it is easier to detect (Van Hulse et al., 2007). Thus, class noise is more often addressed in the literature (Gupta and Gupta, 2019), where several studies analyzed its impact in classification tasks and how to address it (Natarajan et al., 2013; Hendrycks et al., 2018; Liu et al., 2017; Rehbein and Ruppenhofer, 2017; Jindal et al., 2019). In NLP, attribute noise are unintended errors in text, which can come from failures in automatic character recognition processes (Vinciarelli, 2005) or naturally while writing the text in the form of errors in language rules, such as typos, grammatical errors, improper punctuation, irrational capitalization and abbreviations (Agarwal et al., 2007; Contractor et al., 2010; Dey and Haque, 2009; Florian et al., 2010; Michel and Neubig, 2018). In short, noise are unintentional and undesirable errors in the text that can (and should) be eliminated from the data.

Conversely, hard instances are noise-free and cannot be corrected, only eliminated from the data (Smith and Martinez, 2011). In addition, they differ from outliers because their feature representation vectors may be similar to others from regular instances (Smith and Martinez, 2011). Nevertheless, hard instances are more prone to class noise. In fact, Beigman Klebanov and Beigman (2009) defined hard instances in the context of label annotations, under the assumption that items that are easy are reliably annotated, whereas items that are hard display confusion and disagreement among the annotators. Later, Beigman Klebanov and Beigman (2014) showed that the presence of hard instances in the training data misleads the machine learner on easy, clear-cut cases. The definition of Smith et al. (2014) is similar to ours: hard instances are simply those that "should be misclassified" by machine learning methods. The authors introduced hardness measures based on the outputs of an ensemble of classifiers to identify such instances and showed that classifiers are often uncertain about their classes. Following the same idea, Krymolowski (2002) argues that easy instances are correctly classified by all or most classifiers. On the other hand, hard instances are missed by most of them. 
In this work, we propose a human classifier composed by human annotators to identify hard instances. Our definition unifies the ones of Beigman Klebanov and Beigman $(2009,2014)$ and Smith et al. (2014). Similarly to Beigman Klebanov and Beigman $(2009,2014)$, we define hard instances as those in which the human classifier is uncertain or wrong about their true labels. However, different from these studies, which quantify the impact hard instances have on training, our goal is to provide a methodology to quantify the expected amount of hard instances in data and the impact they have on classifiers in production and testing. Also, and similarly to Smith et al. (2014), hard instances are divided into "instances that should be misclassified", which we call discrepant, and "border points", which we call neutral. To the best of our knowledge, we are the first to propose a methodology to characterize and quantify the impact of hard instances in unstructured textual data for polarity classification tasks.

Regarding the effect of hard instances in sentiment and polarity classification tasks, Bermingham and Smeaton (2010) showed that it is easier to classify sentiment in short documents (e.g. tweets) than in longer ones, as short documents have less nonrelevant information. Also, Valdivia et al. (2019) showed that ratings in TripAdvisor reviews are not strongly correlated with sentiment scores given by sentiment analysis methods and proposed a unified index that aggregates both polarities. Barnes et al. (2019) collected a subset of sentences that an ensemble of state-of-the-art sentiment classifiers misclassified and annotated them for 18 linguistic and paralinguistic phenomena, such as negation, sarcasm, among others. In our work, we analyze manually identified hard instances (as opposed to instances misclassified by a machine classifier). As a result, compared to these works, we have a more precise (e.g., a misclassified instance is not necessarily hard) and complete (e.g., not all hard instances are misclassified) ground-truth.

\section{Methodology}

Problem Setting. In this work, we focus on the problem of polarity detection of movie reviews, but all the methods can be applied to any documentlevel polarity classification task. More formally, in a dataset $\mathcal{D}=(X, Y)$ composed by a set of textual movie reviews $X$ and their corresponding binary ratings $Y$, each review $x_{i} \in X$ is associated with a score (or rating) $y_{i} \in Y$ that can be either 0 (positive) or 1 (negative). For the aims of this paper, it is important that $\mathcal{D}$ does not contain any movie reviews that have been explicitly associated with a neutral score by their author, e.g. a score of 50 on Metacritic. By doing this, we isolate hard instances from explicit neutral reviews, avoiding class noise and biases.

Our methodology is composed by a human classifier $f_{H}$, which identifies hard instances, and a machine classifier $f_{M}$, which is tested on hard and regular instances. A classifier is defined as a function $f\left(x_{i}\right)$ that receives a textual movie review $x_{i}$ as input and returns its polarity $\hat{y}_{i} \in\{0,1\}$. We use the human classifier to assign a label $l_{i}$ to a large sample of movie reviews $x_{i}$ to indicate whether $x_{i}$ is a hard instance or not. This label can be one (and only one) of a set $L$ of manually defined labels that indicate that the instance is regular or a type of hard instance. With that, we will be able to quantify the impact of hard instances on machine classifiers and provide explanations about why they occur and how to avoid them in order to improve machine classifiers' accuracy. More specifically, for a machine classifier $f_{M}$ and for all labels $l \in L$, regular included, we will calculate the probabilities $P\left(l_{i}=l \mid y_{i} \neq \hat{y}_{i}\right)$ and $P\left(y_{i}=\hat{y}_{i} \mid l_{i}=l\right)$.

Types of Hard Instances. A strong premise of this work is that the dataset $\mathcal{D}$ has no (or negligible) class noise, i.e., all polarity scores $y_{i} \in Y$ reflect the real opinion of the reviewer. To guarantee that, one needs to construct $\mathcal{D}$ using movie reviews from systems like Metacritic or Rotten Tomatoes, which have well defined meanings for the scores, which are always visible to the reviewers. Thus, every time the polarity of text $x_{i}$ is inconsistent with its true score $y_{i}$, we assume that $x_{i}$ is a hard instance. More specifically, we define two possible hypotheses explaining the hardness of the text $x_{i}$, i.e., two disjoint types of hard instances: (1) the text does not have a clear polarity, namely neutrality, and (2) the text has a clear polarity, but its score $y_{i}$ is the opposite one, namely discrepancy.

A movie review $x_{i}$ is a hard instance of type neutrality when its polarity is not clear. We define three labels for neutral hard instances: mixed (text has mixed opinions), factual (text is purely factual) and contextual (polarity needs context). The mixed label considers reviews that describes both positive and negative points about the movie without having the overall opinion clearly stated. One real exam- 
ple is: "as dumb as the film is, the actors escape relatively unscathed." The factual label defines non-opinionated reviews that describes only facts about the movie, such as: "it is a movie about the World War II and its consequences on the lives of those who survived." The label contextual characterizes reviews where context is needed to understand its polarity, including those containing irony and sarcasm. One real example is: "ultimately, Collin's film is one of forgiveness and that's not the usual way great tragedies end." Finally, the label hard_undefined is given to reviews where the reasons for the lack of polarity are not clear.

The second type of hard instance, namely discrepancy, is given to reviews where the polarity of its text $x_{i}$ is the opposite of the polarity of its score $y_{i}$. For this type, we define a single label: discrepant (polarity of text and score are discrepant). As an example, consider a highly acclaimed movie of a prestigious director, such as Martin Scorsese. Now, consider a reviewer who liked this movie, but unlike the vast majority of critics, found many points that prevent her from giving it a perfect score. Thus, the text will mostly be about its negative points to justify why she is not giving the expected perfect score. Consequently, the text review will appear negative although the score is positive. The following textual review has a clear negative polarity although its score $y_{i}$ is positive: "Thoroughly predictable from start to finish." For more examples, see Tables 4 and 5 in the Appendix.

Human Classifier. A fundamental building block of our methodology is the human classifier $f_{H}$. Human classifiers are often considered to be the upper bound in terms of performance of classification tasks (Stallkamp et al., 2012; Ciresan et al., 2012; Geirhos et al., 2018), which means that when it makes a prediction error, machine classifiers will most likely also miss. Moreover, when a human classifier working on its full capacity makes a mistake, and the class label is correct (i.e. no class noise), then what caused the error is most likely a hard instance (Beigman Klebanov and Beigman, 2014). We use this premise to define the two types of hard instances discussed in the previous section.

In the task of polarity classification of movie reviews, a human classifier mistake can be due to two causes: (C1) the text of the review $x_{i}$ is not clear about its polarity $y_{i}$, or (C2) the score $y_{i}$ is different from the (clearly) perceived polarity of $x_{i}$. In other words, the human classifier $f_{H}$ can be characterized by two binary features when executing this task: whether it is confident about its prediction (F1) and whether it correctly classified the polarity of the review $x_{i}(\mathbf{F} 2)$. Thus, when it makes a mistake, if it was not confident, an error of type $\mathbf{C 1}$ occurs, and when it was confident, an error of type $\mathbf{C} 2$ occurs. The first one (C1) is associated with a hard instance of type neutrality, whereas the second one (C2) is associated with a hard instance of type discrepancy. Also, while the second only occurs when the human classifier $f_{H}$ makes a mistake, the first occurs every time $f_{H}$ is not confident, i.e., it is independent of the prediction $\hat{y}_{i}$.

With the aforementioned rationale, we are ready to propose a well-defined human classifier $f_{H}$ to identify hard instances in movie reviews. First, and in order to construct a robust classifier, $f_{H}$ is an ensemble composed by three independent human classifiers $f_{h 1}, f_{h 2}$ and $f_{h 3}$. In other words, we will use three annotators to label a movie review $x_{i}$ in terms of its polarity and hardness ${ }^{1}$. Each annotator $j \in\{1,2,3\}$ is asked to classify the reviews in two levels. First, they are asked to make a prediction $\hat{y}_{i}^{j}$, i.e., to classify the polarity of the review $x_{i}$ as positive or negative. Second, they are asked to indicate whether they are confident or not about their classification $\hat{y}_{i}^{j}$. We denote the confidence of annotator $j$ on review $x_{i}$ by $c_{i}^{j} \in$ $\{0,1\}$, where $c_{i}^{j}=1$ if $j$ is confident and $c_{i}^{j}=0$ otherwise. If $c_{i}^{j}=0$, then we assume that $x_{i}$ does not contain sufficient information for $j$ to infer its polarity, that is, $x_{i}$ is a hard instance of type neutrality. So, annotator $j$ is asked to choose one label $l_{i}^{j}$ that fits best to the neutrality of $x_{i}$, which can be either mixed, factual or contextual. On the other hand, if $c_{i}^{j}=1$, then $l_{i}^{j}$ is set to regular. This process is illustrated in Figure 1. Of course, each annotator $j$ is independent and cannot see the others' responses.

At the end of this process, for each instance $x_{i}$, we will have three annotation triples $\left(\hat{y}_{i}^{j}, c_{i}^{j}, l_{i}^{j}\right)$, where $\hat{y}_{i}^{j} \in\{0,1\}$ (positive or negative), $c_{i}^{j} \in$ $\{0,1\}$ (not confident or confident) and $l_{i}^{j} \in \mathcal{L}_{N}=$ $\{$ mixed, factual, contextual, regular $\}$. Assuming that all annotators are equally skilled, we aggregate these annotations using majority voting to set the outputs of our human classifier $f_{H}$. For the polarity $\hat{y}_{i}$ and the confidence $c_{i}$, the aggregation is straightforward, as described in Equations 1 and 2:

\footnotetext{
${ }^{1}$ In practice, any number of annotators can be used, including just one.
} 


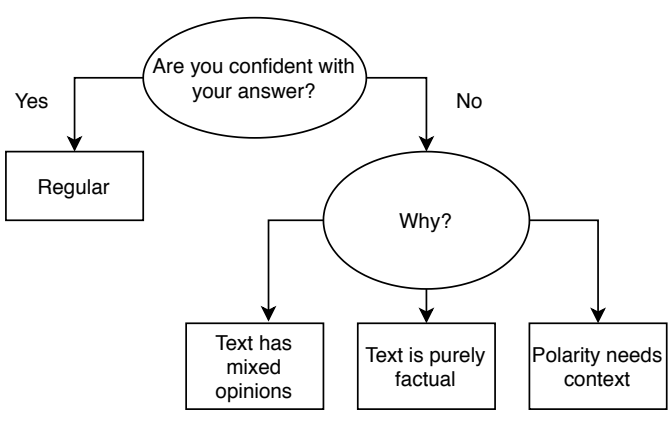

Figure 1: Confidence diagram.

$$
\begin{aligned}
& \hat{y}_{i}= \begin{cases}0 & \text { if } \sum_{j=1}^{3} \hat{y}_{i}^{j} \leq 1 \\
1, & \text { otherwise },\end{cases} \\
& c_{i}= \begin{cases}0 & \text { if } \sum_{j=1}^{3} c_{i}^{j} \leq 1 \\
1, & \text { otherwise. }\end{cases}
\end{aligned}
$$

Setting the final hard instance label $l_{i}$ of review $x_{i}$ is more involved. Let $\mathcal{L}_{i}=\left[l_{i}^{1}, l_{i}^{2}, l_{i}^{3}\right]$ be the list of labels $l_{i}^{j}$ given by the annotators to review $x_{i}$ (e.g. $\mathcal{L}_{1}=[$ mixed, mixed, regular $\left.]\right)$ and $N\left(l, \mathcal{L}_{i}\right)$ the number of elements of $\mathcal{L}_{i}$ that are equal to label $l$ (e.g. $N\left(\right.$ mixed, $\left.\left.\mathcal{L}_{1}\right)=2\right)$. Then, $l_{i}$ is the majority vote if at least two annotators (the majority) gave that label to $x_{i}$ and, if not, $l_{i}$ is set to hard_undefined, indicating no consensus. This process is formally described by Equation 3:

$$
l_{i}= \begin{cases}\arg \max _{l \in \mathcal{L}_{N}} N\left(l, \mathcal{L}_{i}\right) & \text { if } N\left(l, \mathcal{L}_{i}\right) \geq 2 \\ \text { hard_undefined }, & \text { otherwise. }\end{cases}
$$

Finally, when the human classifier is confident about its classification of $x_{i}\left(c_{i}=1\right)$, but it makes a mistake $\left(\hat{y}_{i} \neq y_{i}\right)$, we update the label $l_{i}$ of $x_{i}$ to discrepant. It is easy to see that this update step will be executed only if $l_{i}$ was previously set to regular, i.e., it will not overwrite a neutrality label. Equation 4 defines the discrepancy update step:

$$
l_{i}=\text { discrepant if } \hat{y}_{i} \neq y_{i} \text { and } c_{i}=1 .
$$

\section{Experimental Setup}

Data Set. We collected movie reviews from Metacritic, ${ }^{2}$ which can be authored by regular users and experts, i.e., people working in the movie industry or important communication channels (e.g. The New York Times). In case of experts, the review provided by Metacritic is actually a short summary of the original review and, as we show in Section 5,

\footnotetext{
${ }^{2}$ https:// www metacritic.com/movie
}

this can be a problem for polarity classifiers. Also, each experts review is associated with a score ranging from 0 to 100 , where scores from 0 to 39 are negative, from 40 to 60 are neutral, and from 61 to 100 are positive. Differently, regular users reviews are produced by any person that has an account and are associated with a score ranging from 0 to 10 , where scores between 0 and 3 are negative, between 4 and 6 are neutral, and over 7 are positive. As previously mentioned, the meaning of each rating is clearly conveyed to users in the Metacritic website. Thus, class noise and biases should be rare in the dataset.

In total, we collected 415,867 reviews for 8,170 different movies, where 227,348 of those are from regular users and 188, 519 from experts. Our data collection was executed using the following steps. First, we collected the most popular experts from the website, as provided by Metacritic. Then, we generated a list of all movies reviewed by the top 10 experts. From this list, which contains 8,170 movies, we collected all reviews from experts and regular users that were posted until August, 2018. For the purpose of this work, we avoided reviews that do not have a clear polarity (neutral reviews), i.e., we only considered positive and negative reviews. Hence, we selected a clean and unambiguous dataset. Reviews from experts are usually shorter than from regular users, containing an average of 26 words (std. dev. of 13) against an average of 100 words (std. dev. of 129) for reviews by regular users. In addition, we observed that experts use a more elaborate language. Because of these differences, we will condition our analyses on the type of user (experts or regular users) and score polarity (positive or negative).

Machine Classifiers. To evaluate the impact of hard instances on machine classifiers, we selected three state-of-the-art models with reported success in the task of polarity detection of movie reviews: BERT (Devlin et al., 2019), CNN-GRU (Wang et al., 2016) and C-LSTM (Zhou et al., 2015). CLSTM utilizes a Convolutional Neural Network (CNN) to extract a sequence of higher-level phrase representations, which are then fed into a Long Short-Term Memory (LSTM) unit to obtain the sentence representation. CNN-GRU connects a character-aware CNN with a character-aware Gated Recurrent Unit (GRU) to learn long sequence semantics. These two networks are initialized with pre-trained Word2vec vectors from Google News 
Dataset and have their final representations connected to a dense layer. BERT uses a masked language model (MLM) to pre-train deep bidirectional representations from unlabeled text that considers both the left and right context of sentences and words. In this work, we used an architecture composed by BERT embeddings pre-trained with data from Wikipedia connected with a dense layer. For all architectures, the output $\hat{y}_{i}$ is given by a sigmoid function. For implementation and code details, please see the Appendix.

\section{Results}

\subsection{Number of Hard Instances}

The first question we need to answer is: how many hard instances exist in movie reviews? In the context of our Metacritic dataset $\mathcal{D}$, the answer to this question can be influenced by two factors: (1) the type of user and (2) the polarity of their rating. Thus, the following results are conditioned on whether the authors are experts or regular users and whether the reviews are positive or negative. Because of that, we sampled a collection $D_{H}$ of 800 movie reviews from $\mathcal{D}$ that is both balanced in terms of user type and score polarity, i.e., this collection has 200 reviews for each of the four combinations of user type and score polarity.

In order to quantify the number of hard instances in $D_{H}$, we use our proposed human classifier $f_{H}$ described in Section 3 to label every review $x_{i} \in D_{H}$. Recall that $f_{H}$ assigns a polarity $\hat{y}_{i} \in\{$ positive, negative $\}$ to $x_{i}$ and, more important to our purpose here, a label $l_{i}$, which can be either regular (instance is not a hard instance), discrepant (the polarity of the text is different from the score polarity), or one of the four neutrality labels: mixed (text has mixed opinions), factual (text is purely factual), contextual (polarity needs context) and hard_undefined (reasons are unclear). Also, let $u_{i} \in\{$ expert, regular user $\}$ be the user type of the author of review $x_{i}$. Our goal with the following results is to estimate the probability $P\left(l_{i}=l \mid y_{i}=y, u_{i}=u\right)$ for the four combinations of score polarity $y$ and user type $u$.

In Table 1, we show the number and proportion of movie reviews that are or are not hard instances for experts. From the 400 labeled reviews, almost one quarter (92) are hard instances. From those, note that neutral reviews are more common than discrepant ones, but while the first is equally present in both positive and negative reviews, dis-

\begin{tabular}{|l|c|c|c|}
\hline label $\left(l_{i}\right)$ & positive & negative & total \\
\hline \hline \multicolumn{4}{|c|}{ experts } \\
\hline regular & $146(36.5 \%)$ & $162(40.5 \%)$ & $\mathbf{7 7} \%$ \\
\hline discrepant & $20(5 \%)$ & $3(0.8 \%)$ & $\mathbf{5 . 8} \%$ \\
\hline neutral & $34(8.5 \%)$ & $35(8.8 \%)$ & $\mathbf{1 7 . 3} \%$ \\
\hline \hline mixed & $10(2.5 \%)$ & $7(1.8 \%)$ & $\mathbf{4 . 3} \%$ \\
\hline factual & $14(3.5 \%)$ & $3(0.8 \%)$ & $\mathbf{4 . 3} \%$ \\
\hline contextual & $7(1.8 \%)$ & $20(5 \%)$ & $\mathbf{6 . 8} \%$ \\
\hline undefined & $3(0.8 \%)$ & $5(1.3 \%)$ & $\mathbf{2} \%$ \\
\hline \multicolumn{4}{|c|}{ regular users } \\
\hline regular & $177(44.3 \%)$ & $187(46.8 \%)$ & $\mathbf{9 1} \%$ \\
\hline discrepant & $3(0.8 \%)$ & $2(0.5 \%)$ & $\mathbf{1 . 3} \%$ \\
\hline neutral & $20(5 \%)$ & $11(2.8 \%)$ & $\mathbf{7 . 8} \%$ \\
\hline \hline mixed & $16(4 \%)$ & $7(1.8 \%)$ & $\mathbf{5 . 8} \%$ \\
\hline factual & $1(0.3 \%)$ & $2(0.5 \%)$ & $\mathbf{0 . 8} \%$ \\
\hline contextual & $0(0 \%)$ & $1(0.3 \%)$ & $\mathbf{0 . 3} \%$ \\
\hline undefined & $3(0.8 \%)$ & $1(0.3 \%)$ & $\mathbf{1} \%$ \\
\hline
\end{tabular}

Table 1: Number of hard instances in reviews.

crepant instances are significantly more present in positive reviews. In such cases, the author gave a positive score to the movie, but its review demonstrates the opposite sentiment. This often occurs when the expert is using the review to justify a good, but far from perfect score, to a critically acclaimed movie. As for the neutral reviews, the most predominant type is contextual $(6.8 \%)$, followed by mixed (4.3\%) and factual (4.3\%). Also, contextual instances are more common in negative reviews, when experts often use figures of speech (e.g. irony) together with external knowledge to create humour. Finally, factual instances are more present in positive reviews, where the experts simply describe some characteristic of the movie that impressed them without explicitly saying that.

Also, in Table 1 we show the number and proportion of movie reviews that are or are not hard instances for regular users. First, note that the number of reviews that are hard instances significantly decreased in comparison with the ones written by experts. From the 400 labeled reviews, only $36(9 \%)$ are hard instances, of which 31 are neutral and only 5 are discrepant. Different from what was verified for experts, the most predominant label for regular users was mixed, which occurred significantly more in positive reviews. For the other labels, their occurrences were fairly balanced between negative and positive reviews. We observed that regular users use a much more direct and simple language to state their opinions than experts. Because of that, most of the hard instances are concentrated in cases where the author lists both the negative and positive aspects of the movie without stating their final opinions about the movie, which 
is the definition of mixed.

A note about the human classifier. Because we used three human annotators in $f_{H}$ and a majority vote function, only two annotators were used initially. The third annotator was called to classify $x_{i}$ if, and only if, the first two had any kind of disagreement, i.e., a disagreement regarding the polarity $y_{i}$, the confidence $c_{i}$, or label $l_{i}$. For the first two annotators, they agreed on $91.13 \%$ of the polarity scores, on $90.5 \%$ of their confidence levels and on $88 \%$ of their labels. Regarding the third annotator, only $1.5 \%$ of the instances were not in total agreement with at least one of the other annotators. The Cohen's kappa coefficient for the first two annotators was 0.82 in relation to polarity scores, 0.58 regarding their confidence levels and 0.49 regarding their attribute noise labels.

\subsection{Impact of Hard Instances}

In this section, we quantify the impact of hard instances in machine classifiers. Also, by putting these results in perspective with what was achieved by the human classifier, we hope to provide an accurate assessment on how distant machine classifiers are with respect to human performance. We guide our analyses by the following questions:

1. What are the probabilities of a correct and a misclassification given the label $l$ ? In other words, we want to estimate the probabilities $P\left(\hat{y}_{i}=y_{i} \mid l_{i}=l\right)$ and $P\left(\hat{y}_{i} \neq y_{i} \mid l_{i}=l\right)$ for all labels $l \in L$.

2. What are the probabilities of label $l$ given that the classifier was correct and that it made a mistake? In other words, we want to estimate the probabilities $P\left(l_{i}=l \mid \hat{y}_{i} \neq y_{i}\right)$ and $P\left(l_{i}=l \mid \hat{y}_{i}=y_{i}\right)$ for all labels $l \in L$.

To address these questions, we test the three classifiers described in Section 4 in the labeled dataset $D_{H}$ (see Section 5.1), which contains 800 reviews. Because this dataset is completely balanced, we created two balanced training datasets, one containing solely reviews from experts, namely $D_{T}^{\text {experts }}$, and another containing solely reviews from regular users, namely $D_{T}^{\text {users }}$. Each dataset contains 8, 796 reviews, 4, 398 of each polarity. Again, this dataset is solely used to train the machine classifiers. Because these classifiers are sensitive to initialization parameters, we trained and tested them 5 times and the corresponding error bars are shown in Figure 2. Finally, recall that $y_{i}$ refers to the author's original polarity score (gold polarity) and $\hat{y}_{i}$ refers to the polarity predicted by the classifiers, including the human classifier.

Figure 2 shows the classification error (with their respective error bars) for all classifiers in $D_{H}$. The classification error is simply the proportion of instances that were misclassified. Each bar is also colored according to the labels' proportion in the misclassified instances. For each classifier, the left (right) bar shows the error with respect to positive (negative) instances. In general, the human classifier was the one that achieved the smallest error, followed by BERT and C-LSTM. Also, the errors are always higher for experts, as these reviews have significantly less words (see Section 4) and more hard instances (see Section 5.1). The latter is also one of the main reasons for the error being almost always higher for positive instances than for negative instances. For expert reviews, while negative instances always have more regular instances, positive instances have almost twice more hard instances, particularly discrepant ones. For regular user reviews, positive instances also have more hard instances, but the difference in terms of neutral reviews is more significant. Note that, for both user types, this difference in the instances misclassified by the human classifier is striking.
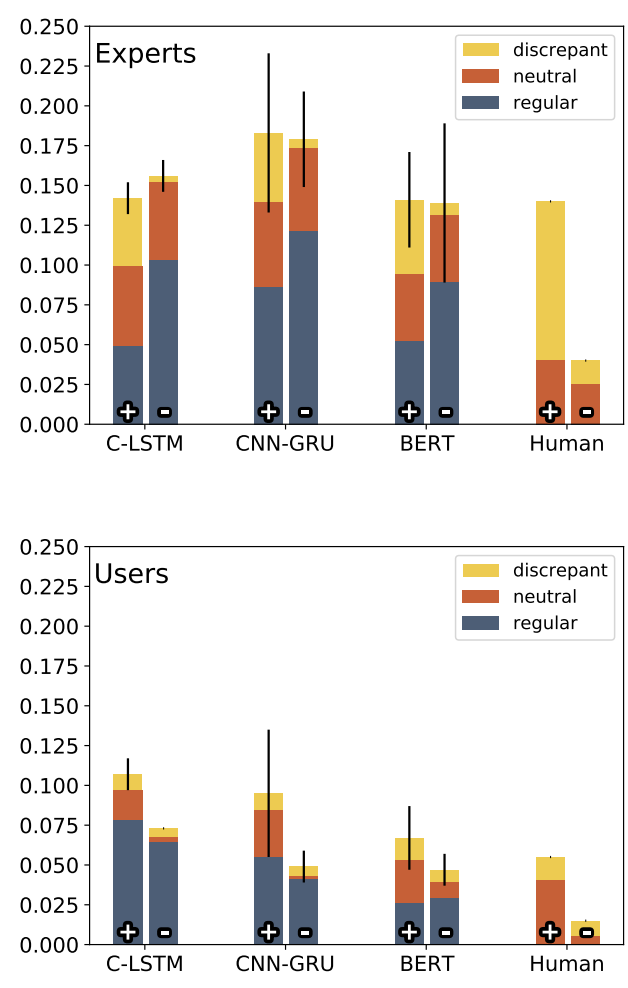

Figure 2: Classification error for all classifiers. 
For a more precise assessment of the impact of hard instances, we show in Table 2 the accuracy of the classifiers considering instances of each label separately. In other words, these results provide estimates for the probabilities of our first question, $P\left(\hat{y}_{i}=y_{i} \mid l_{i}=l\right)$ and $P\left(\hat{y}_{i} \neq y_{i} \mid l_{i}=l\right)$. First, note that for all classifiers the accuracy significantly degrades in neutral instances and get even worse in discrepant instances. Recall that a discrepant review is a review where the human classifier was sure about its polarity, but the originally assigned polarity is the opposite. Thus, by definition, the human classifier accuracy on discrepant reviews is zero. For neutral instances, the human classifier always outperforms the machine classifiers. However, the machine classifiers are not always tricked by discrepant reviews as the human classifier is, although their performances are not better than a coin toss. Considering the specific neutral labels, note that BERT achieves human level performance for contextual, which is coherent with the nature of this classifier, given that its embeddings are supposed to carry much more contextual information in comparison with the embeddings used in C-LSTM and CNN-GRU. The most inconclusive results refer to hard_undefined, which is also the label with the least instances, 12 out of 800 .

\begin{tabular}{|l|c|c|c|c|}
\cline { 2 - 5 } \multicolumn{1}{c|}{} & C-LSTM & CNN-GRU & BERT & Human \\
\hline regular & 0.91 & 0.91 & 0.94 & $\mathbf{1}$ \\
\hline discrepant & $\mathbf{0 . 5 5}$ & 0.52 & 0.45 & 0 \\
\hline neutral & 0.76 & 0.73 & 0.76 & $\mathbf{0 . 7 8}$ \\
\hline \hline mixed & 0.75 & 0.72 & $\mathbf{0 . 7 6}$ & 0.75 \\
\hline factual & 0.78 & 0.77 & 0.71 & $\mathbf{0 . 8 0}$ \\
\hline contextual & 0.67 & 0.64 & $\mathbf{0 . 7 9}$ & $\mathbf{0 . 7 9}$ \\
\hline undefined & $\mathbf{0 . 9 7}$ & 0.90 & 0.77 & 0.83 \\
\hline
\end{tabular}

Table 2: Accuracy of the classifiers considering only instances of a particular label.

To answer our second question, related to the probabilities $P\left(l_{i}=l \mid \hat{y}_{i} \neq y_{i}\right)$ and $P\left(l_{i}=\right.$ $\left.l \mid \hat{y}_{i}=y_{i}\right)$, we sample an additional dataset $D_{H}^{\text {error }}$ to be labeled by our human classifier $f_{H}$. First, we run the BERT classifier, which was the one that achieved the best results, on two new balanced sets of reviews extracted from $\mathcal{D}$, one containing 2,752 reviews from experts and the other 2,752 reviews from regular users. Again, we used the same BERT classifiers that were trained for generating the results in Figure 2, one for each user type. After running BERT, we construct $D_{H}^{\text {error }}$ by sampling 100 misclassified and 100 correctly classified instances authored by each user type, for a total of
400 reviews. Then, we run $f_{H}$ on $D_{H}^{\text {error }}$ to have a more accurate estimate of $P\left(l_{i}=l \mid \hat{y}_{i} \neq y_{i}\right)$ and $P\left(l_{i}=l \mid \hat{y}_{i}=y_{i}\right)$.

\begin{tabular}{|l|c|c|}
\hline label $\left(l_{i}\right)$ & $\hat{\mathbf{y}}_{\mathbf{i}}=\mathbf{y}_{\mathbf{i}}$ & $\hat{\mathbf{y}}_{\mathbf{i}} \neq \mathbf{y}_{\mathbf{i}}$ \\
\hline \hline \multicolumn{3}{|c|}{ experts } \\
\hline regular & $96(78 \%)$ & $28(36 \%)$ \\
\hline discrepant & $1(1 \%)$ & $19(25 \%)$ \\
\hline neutral & $26(21 \%)$ & $30(39 \%)$ \\
\hline \hline mixed & $10(8 \%)$ & $9(11 \%)$ \\
\hline factual & $6(5 \%)$ & $3(4 \%)$ \\
\hline contextual & $8(6 \%)$ & $11(14 \%)$ \\
\hline undefined & $2(2 \%)$ & $7(9 \%)$ \\
\hline \hline \multicolumn{3}{|c|}{ regular users } \\
\hline regular & $111(86 \%)$ & $31(44 \%)$ \\
\hline discrepant & $2(2 \%)$ & $14(19 \%)$ \\
\hline neutral & $16(12 \%)$ & $26(37 \%)$ \\
\hline \hline mixed & $13(10 \%)$ & $15(21 \%)$ \\
\hline factual & $0(0 \%)$ & $1(1 \%)$ \\
\hline contextual & $1(1 \%)$ & $6(8 \%)$ \\
\hline undefined & $1(1 \%)$ & $5(6 \%)$ \\
\hline
\end{tabular}

Table 3: Percentage of labels in correct $\left(\hat{y}_{i}=y_{i}\right)$ and incorrect $\left(\hat{y}_{i} \neq y_{i}\right)$ predictions by BERT.

Table 3 shows the percentages of each label for correctly and incorrectly classified instances, which provide estimates for the probabilities of $P\left(l_{i}=l \mid \hat{y}_{i} \neq y_{i}\right)$ and $P\left(l_{i}=l \mid \hat{y}_{i}=y_{i}\right)$. For both experts and regular users, it is much more likely to find neutral and discrepant reviews in misclassified instances. In other words, one easy way to find hard instances in movie reviews is to run BERT and sample from misclassified instances. Our estimates for the probabilities of finding a misclassified hard instance is 0.64 for experts and 0.56 for regular users. In other words, more than $50 \%$ of our sampled misclassified instances are hard instances. Recall from Table 1 that we found only $23 \%$ of hard instances in reviews from experts and only $9 \%$ in reviews from regular users in our first balanced sample $D_{H}$. The most striking difference is for discrepant reviews, where the number of instances increased by one order of magnitude in misclassified instances. Regarding the neutral labels, our results reveal that we are at least twice as likely to find contextual instances in misclassified expert reviews and mixed instances in misclassified regular users reviews. Therefore, to find hard instances with high probability, we propose to train and run BERT in the data (without filtering anything) and, from the misclassified instances, run the human classifier to identify them.

We investigated misclassified regular instances and found two patterns that explain the errors. First, reviews that have positive and negative points, but 
where humans can easily identify what side has the most weight. Second, reviews that have some "irony" that is clear to humans, but is created using words with the opposite polarity of the final score $y_{i}$. For examples, see Table 6 in the Appendix. We conjecture that these instances can be correctly classified with extra training and more modern (and complex) architectures. On the other hand, we feel that dealing with hard instances is not that simple, where more guided and focused approaches are probably needed, such as the one proposed by Valdivia et al. (2019). They proposed an approach to combine reviews with scores for an aggregated polarity, which can be a good idea to deal with hard instances.

Overview of our results. Our first goal was to quantify the expected amount of hard instances in misclassifications, which is $\approx 56 \%$ for regular users and $\approx 64 \%$ for experts. Note that even though the reviews for these users are intrinsically different, the values are similar. The second goal was to quantitatively show how different the two types of hard instances are. Table 1 shows that neutral instances are common, and Table 3 shows they might have a significant presence even in correctly classified instances. Contrastingly, discrepant instances are rare, particularly among correctly classified instances. Given that our ultimate goal was to quantify and explain the reasons behind misclassifications, from Table 3 we can say that most of the mistakes $(\approx 60 \%)$ occur because of neutral $(\approx 38 \%)$ and discrepant $(\approx 22 \%)$ instances.

\section{Conclusion}

In this work, we propose a methodology to characterize, quantify and measure the impact of hard instances in the task of polarity classification of movie reviews. We characterized such instances into two disjoint categories: neutrality and discrepancy. We provided empirical evidence about the need to pay attention to such instances, as they are much harder to be classified, for both machine and human classifiers.

The main hypothesis of this work is that hard instances can make polarity classifiers fail. To demonstrate this hypothesis, we provided two well defined types of hard instances, which are based on human reasoning, and a methodology to find them in labeled data. With that, one can quantify how many instances of those types there are in their data, which can shed light on why and when classifiers fail. We collected a noise-free (no class noise) and well separated (no neutral polarity) dataset and showed that even in such a dataset most of the mistakes made by a state of the art classifier, namely BERT, are in our defined hard instances. Observe in Table 3 that more than $50 \%$ of our sampled misclassified instances are hard instances (discrepant or neutral).

Our methodology works for every type of supervised classification task. Because our proposed labels are defined from the perspective of a classifier fully capable of human-reasoning, they are easy to interpret and can be generalized to every classification task (e.g. polarity, image, song genre, topic) that humans are able to do. After employing our methodology, it will be possible to differentiate mistakes that come from hard instances, which are those even humans cannot classify with confidence (or at all), and mistakes that could be solved by improving the classifier architecture. In short, our proposed methodology can help quantify and explain why classifiers are making mistakes.

We made the dataset containing the labels publicly available ${ }^{3}$ so it can be used as a standard benchmark for robustness to hard instances in polarity classification tasks, and to potentially foster research on models, datasets and evaluation metrics tailored for this problem.

\section{Acknowledgements}

This work is supported by the authors' individual grants from FAPEMIG, CAPES and CNPq. We also thank all the reviewers for their thoughtful comments which helped to improve this work.

\section{References}

S. Agarwal, S. Godbole, D. Punjani, and S. Roy. 2007. How much noise is too much: A study in automatic text classification. In Seventh IEEE International Conference on Data Mining (ICDM 2007), pages 312.

Ravi Arunachalam and Sandipan Sarkar. 2013. The new eye of government: Citizen sentiment analysis in social media. In Proceedings of the IJCNLP 2013 workshop on natural language processing for social media (SocialNLP), pages 23-28.

Jeremy Barnes, Lilja Øvrelid, and Erik Velldal. 2019. Sentiment Analysis Is Not Solved! Assessing and

\footnotetext{
${ }^{3}$ https://github.com/karenstemartins/
} NAACL 2021 
Probing Sentiment Classification. In Proceedings of the 2019 ACL Workshop BlackboxNLP: Analyzing and Interpreting Neural Networks for NLP, pages 12-23, Stroudsburg, PA, USA. Association for Computational Linguistics.

Beata Beigman Klebanov and Eyal Beigman. 2009. From Annotator Agreement to Noise Models. Computational Linguistics, 35(4):495-503.

Beata Beigman Klebanov and Eyal Beigman. 2014. Difficult cases: From data to learning, and back. 52nd Annual Meeting of the Association for Computational Linguistics, ACL 2014 - Proceedings of the Conference, 2:390-396.

Adam Bermingham and Alan F. Smeaton. 2010. Classifying sentiment in microblogs: Is brevity an advantage? In Proceedings of the 19th ACM International Conference on Information and Knowledge Management, CIKM '10, page 1833-1836, New York, NY, USA. Association for Computing Machinery.

Marc Cheong and Vincent C S Lee. 2011. A microblogging-based approach to terrorism informatics: Exploration and chronicling civilian sentiment and response to terrorism events via Twitter. Information Systems Frontiers, 13(1):45-59.

D. Ciresan, U. Meier, and J. Schmidhuber. 2012. Multicolumn deep neural networks for image classification. In 2012 IEEE Conference on Computer Vision and Pattern Recognition, pages 3642-3649. IEEE.

Danish Contractor, Tanveer A. Faruquie, and L. Venkata Subramaniam. 2010. Unsupervised cleansing of noisy text. In Coling 2010: Posters, pages 189-196. Coling 2010 Organizing Committee.

Jacob Devlin, Ming-Wei Chang, Kenton Lee, and Kristina Toutanova. 2019. BERT: Pre-training of deep bidirectional transformers for language understanding. In Proceedings of the 2019 Conference of the North American Chapter of the Association for Computational Linguistics: Human Language Technologies, Volume 1 (Long and Short Papers), pages 4171-4186. Association for Computational Linguistics.

Lipika Dey and S. K. Mirajul Haque. 2009. Studying the effects of noisy text on text mining applications. In Proceedings of The Third Workshop on Analytics for Noisy Unstructured Text Data, AND '09, page 107-114, New York, NY, USA. Association for Computing Machinery.

Mohamed Elaraby and Muhammad Abdul-Mageed. 2018. Deep models for Arabic dialect identification on benchmarked data. In Proceedings of the Fifth Workshop on NLP for Similar Languages, Varieties and Dialects (VarDial 2018), pages 263-274. Association for Computational Linguistics.
Radu Florian, John Pitrelli, Salim Roukos, and Imed Zitouni. 2010. Improving mention detection robustness to noisy input. In Proceedings of the 2010 Conference on Empirical Methods in Natural Language Processing, pages 335-345, Cambridge, MA. Association for Computational Linguistics.

Benoit Frenay and Michel Verleysen. 2014. Classification in the Presence of Label Noise: A Survey. IEEE Transactions on Neural Networks and Learning Systems, 25(5):845-869.

Robert Geirhos, Carlos R. M. Temme, Jonas Rauber, Heiko H. Schütt, Matthias Bethge, and Felix A. Wichmann. 2018. Generalisation in humans and deep neural networks. In S. Bengio, H. Wallach, H. Larochelle, K. Grauman, N. Cesa-Bianchi, and R. Garnett, editors, Advances in Neural Information Processing Systems 31, pages 7538-7550. Curran Associates, Inc.

Andrew B Goldberg, Xiaojin Zhu, and Stephen Wright. 2007. Dissimilarity in graph-based semi-supervised classification. In Artificial Intelligence and Statistics, pages 155-162.

Lin Gui, Yu Zhou, Ruifeng Xu, Yulan He, and Qin Lu. 2017. Learning representations from heterogeneous network for sentiment classification of product reviews. Knowledge-Based Systems, 124:34-45.

Shivani Gupta and Atul Gupta. 2019. Dealing with noise problem in machine learning data-sets: A systematic review. Procedia Computer Science, 161:466 - 474. The Fifth Information Systems International Conference, 23-24 July 2019, Surabaya, Indonesia.

Dan Hendrycks, Mantas Mazeika, Duncan Wilson, and Kevin Gimpel. 2018. Using trusted data to train deep networks on labels corrupted by severe noise. In Advances in neural information processing systems, pages 10456-10465.

Ishan Jindal, Daniel Pressel, Brian Lester, and Matthew Nokleby. 2019. An effective label noise model for dnn text classification. In Proceedings of the 2019 Conference of the North American Chapter of the Association for Computational Linguistics, pages 3246-3256. Association for Computational Linguistics.

Yuval Krymolowski. 2002. Distinguishing easy and hard instances. In COLING-02: The 6th Conference on Natural Language Learning 2002 (CoNLL2002).

Tianyu Liu, Kexiang Wang, Baobao Chang, and Zhifang Sui. 2017. A Soft-label Method for Noisetolerant Distantly Supervised Relation Extraction. In Proceedings of the 2017 Conference on Empirical Methods in Natural Language Processing, pages 1790-1795. Association for Computational Linguistics. 
Ismini Lourentzou, Kabir Manghnani, and ChengXiang Zhai. 2019. Adapting sequence to sequence models for text normalization in social media. Proceedings of the International AAAI Conference on Web and Social Media, 13(01):335-345.

Scott M Lundberg and Su-In Lee. 2017. A unified approach to interpreting model predictions. In I. Guyon, U. V. Luxburg, S. Bengio, H. Wallach, R. Fergus, S. Vishwanathan, and R. Garnett, editors, Advances in Neural Information Processing Systems 30, pages 4765-4774. Curran Associates, Inc.

Paul Michel and Graham Neubig. 2018. MTNT: A Testbed for Machine Translation of Noisy Text. In Proceedings of the 2018 Conference on Empirical Methods in Natural Language Processing, pages 543-553.

Nagarajan Natarajan, Inderjit S. Dhillon, Pradeep Ravikumar, and Ambuj Tewari. 2013. Learning with noisy labels. In Proceedings of the 26th International Conference on Neural Information Processing Systems - Volume 1, NIPS'13, page 1196-1204. Curran Associates Inc.

Federico Alberto Pozzi, Elisabetta Fersini, Enza Messina, and Bing Liu. 2016. Sentiment analysis in social networks. Morgan Kaufmann.

Ines Rehbein and Josef Ruppenhofer. 2017. Detecting annotation noise in automatically labelled data. In Proceedings of the 55th Annual Meeting of the Association for Computational Linguistics (Volume 1: Long Papers), pages 1160-1170. Association for Computational Linguistics.

Filipe N Ribeiro, Matheus Araújo, Pollyanna Gonçalves, Marcos André Gonçalves, and Fabrício Benevenuto. 2016. SentiBench - a benchmark comparison of state-of-the-practice sentiment analysis methods. EPJ Data Science, 5(1):23.

Michael R. Smith and Tony Martinez. 2011. Improving classification accuracy by identifying and removing instances that should be misclassified. In The 2011 International Joint Conference on Neural Networks, pages 2690-2697. IEEE.

Michael R. Smith, Tony Martinez, and Christophe Giraud-Carrier. 2014. An instance level analysis of data complexity. Machine Learning, 95(2):225256.

J. Stallkamp, M. Schlipsing, J. Salmen, and C. Igel. 2012. Man vs. computer: Benchmarking machine learning algorithms for traffic sign recognition. $\mathrm{Neu}$ ral Networks, 32:323-332.

Ana Valdivia, Emiliya Hrabova, Iti Chaturvedi, M. Victoria Luzón, Luigi Troiano, Erik Cambria, and Francisco Herrera. 2019. Inconsistencies on TripAdvisor reviews: A unified index between users and Sentiment Analysis Methods. Neurocomputing, 353:316.
Jason D. Van Hulse, Taghi M. Khoshgoftaar, and Haiying Huang. 2007. The pairwise attribute noise detection algorithm. Knowledge and Information Systems, 11(2):171-190.

A. Vinciarelli. 2005. Noisy text categorization. IEEE Transactions on Pattern Analysis and Machine Intelligence, 27(12):1882-1895.

Xingyou Wang, Weijie Jiang, and Zhiyong Luo. 2016. Combination of convolutional and recurrent neural network for sentiment analysis of short texts. In Proceedings of COLING 2016, the 26th International Conference on Computational Linguistics: Technical Papers, pages 2428-2437. The COLING 2016 Organizing Committee.

Yongfeng Zhang. 2015. Incorporating Phrase-level Sentiment Analysis on Textual Reviews for Personalized Recommendation. In Proceedings of the Eighth ACM International Conference on Web Search and Data Mining - WSDM '15, pages 435440. ACM Press.

Chunting Zhou, Chonglin Sun, Zhiyuan Liu, and Francis C. M. Lau. 2015. A C-LSTM neural network for text classification. CoRR, abs/1511.08630.

Xingquan Zhu and Xindong Wu. 2004. Class Noise vs. Attribute Noise: A Quantitative Study. Artificial Intelligence Review, 22(3):177-210.

\section{A Appendix}

\section{A.1 Examples of Reviews}

Tables 4 and 5 show some examples of hard instances labeled by our human classifier. All the code and data is publicly available at https://github.com/ karenstemartins/NAACL2021.

\begin{tabular}{|c|c|}
\hline $\begin{array}{c}\text { class label } \\
\left(l_{i}\right)\end{array}$ & Example \\
\hline discrepant & $\begin{array}{l}\text { "Figgis's film doesn't match its reach." } \\
\text { (Positive) }\end{array}$ \\
\hline mixed & "Pleasant but dull formula film." (Negative) \\
\hline factual & $\begin{array}{l}\text { "Without trivializing the disease, the film } \\
\text { challenges AIDS' stigma (albeit for } \\
\text { heterosexuals) at a moment when it was still } \\
\text { considered a death sentence." (Positive) }\end{array}$ \\
\hline contextual & $\begin{array}{l}\text { "Disheveled tripe pieced together } \\
\text { with the good intentions." (Negative) }\end{array}$ \\
\hline undefined & $\begin{array}{l}\text { "More interesting as history, re-written, than } \\
\text { as the moral parable this true story became." } \\
\text { (Positive) }\end{array}$ \\
\hline
\end{tabular}

Table 4: Examples of hard instances from experts.

\section{A.2 Regular Reviews}

Table 6 shows real examples of misclassified regular reviews with their original polarities given by their authors. The first and last review contain 


\begin{tabular}{|c|c|}
\hline $\begin{array}{c}\text { class label } \\
\left(l_{i}\right)\end{array}$ & \multicolumn{1}{c|}{ Example } \\
\hline discrepant & $\begin{array}{c}\text { "The actors try their best with the lines they } \\
\text { are given, but the "movie about a real bank } \\
\text { robbery" is on auto-pilot most of the time. } \\
\text { It greatly resembles a 70's film by letting } \\
\text { the characters drive the story. As a result } \\
\text { there's a lot of dialog. But its not very } \\
\text { interesting dialog. It is an instantly } \\
\text { forgettable film." (Positive) }\end{array}$ \\
\hline mixed & $\begin{array}{c}\text { "I think the director did an incredible job. I } \\
\text { loved the way it was shot. The scifi world } \\
\text { they created was also awesome. But I think } \\
\text { the story was way too subtle and wasn't } \\
\text { clear enough." (Positive) }\end{array}$ \\
\hline factual & $\begin{array}{c}\text { "(...) The 1953 film about a provincial old } \\
\text { couple's pilgrimage to the big city provokes } \\
\text { sympathy for the mother and father, who } \\
\text { are so frail, so gentle, and yet are treated so } \\
\text { badly by their urbanized son and daughter. } \\
\text { (...)" (Positive) }\end{array}$ \\
\hline contextual & $\begin{array}{l}\text { "Only go if you're interested in seeing } \\
\text { Bening's new facelift." (Negative) }\end{array}$ \\
\hline undefined & $\begin{array}{l}\text { "Wow, can't believe the critics on this one." } \\
\text { (Positive) }\end{array}$ \\
\hline
\end{tabular}

Table 5: Examples of hard instances from regular users.

some "irony" that is clear to humans, but they are created using words with their opposite polarity of the final score. The second review contains positive and negative points of the movie, but humans can easily identify what side has the most weight.

\begin{tabular}{|c|}
\hline Examples \\
\hline "Michael Bay may think that special effects can substitute \\
for good acting and a good story, but that does not \\
fly around here." (Negative) \\
\hline "From the first moment of Superman till the very end \\
scene Lex luthor this is a true comic book movie \\
adaption. True there are few CGI errors, but "Nothing \\
is perfect in this world" and this is just a movie." \\
(Positive) \\
\hline "The trailer was promising to me; I expected it to be a \\
really good movie, but instead it was "meh". I didn't \\
really like Cruz; it was heartwarming how Lightning \\
McQueen made a tribute to Doc at the end, but the \\
trailer made it seem action packed; it wasn't \\
as good as I expected." (Negative)
\end{tabular}

Table 6: Examples of misclassified regular reviews.

We further investigate these patterns by using SHAP (Lundberg and Lee, 2017), which is a game theoretic approach to explain the output of deep learning models and designed to understand the most important words for the machine classifiers. Figure 3 shows the result for the last review in Table 6 . The words are plotted in descending order according with their importance. Note that, all listed words have a positive polarity when they are analyzed separately. As a result, their combination contributes to the classifier misclassify the review.

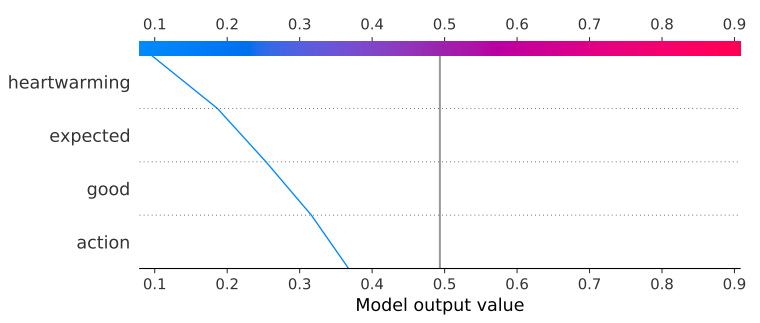

Figure 3: SHAP plot for the last review in Table 6.

\section{A.3 Machine Classifier}

\section{A.3.1 Third Part Material}

The code of the three machine classifiers used in this work are publicly available in the Internet. CNN-GRU and BERT were published by their authors and C-LSTM by researchers who used this method in their work (Elaraby and Abdul-Mageed, 2018). We made small modifications in the codes so they are able to process our movie reviews data. We also created a log module to register all the results and changed the final output layer to a sigmoid function, since our problem is a binary classification. We also made BERT use the Keras library just to facilitate our comparisons, but this is not a necessary step to reproduce our results. The link to each repository is listed bellow:

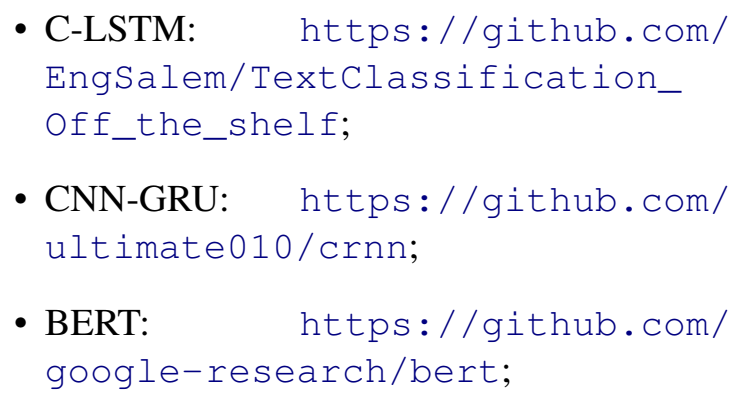

\section{A.3.2 Model Training}

To train the machine classifiers, we randomly generated two balanced partitions of our data with the same size, one for experts and other for regular users. Each training dataset contains 4,398 positive and 4,398 negative reviews, for a total of 8,796 reviews. It is important to note that these datasets do not contain any review labeled by the human classifier. After that, we performed a 5-fold cross-validation to choose the best hyperparameters for our data. The set of hyperparameter configurations we tested were the same used in the original articles (Wang et al., 2016), (Zhou et al., 2015) and (Devlin et al., 2019). Since the BERT architecture 
is very simple, it has only a single hyperparameter, the batch size, for which we tested values of 16,32 and 64 . For C-LSTM, we tested layers with 100, 150 and 200 filters, and filters of size 2, 3 and 4, memory dimensions of size 100, 150 and 200, and batch size of 16, 32 and 64 . Finally, for CNN-GRU, we tested layers with 100 and 200 filters, filters of size 3 and 4, GRU dimensionality of 100 and 150, pool sizes of 2 and 3 , and batch sizes of 16 and 32 . To run our experiments, we use a computer with the following configuration: $32 \mathrm{RAM}$, Intel Core i7 CPU 3.40 GHz and NVIDIA GeForce GTX GPU.

After executing cross-validation, we selected the best hyperparameters for each architecture and type of users comparing their F1-Score. We use 256 words for models trained with expert data and 512 for those trained with regular user data in all architectures. BERT achieved the best results using a batch size of 16 for both user types. For experts, C-LSTM uses a batch size of 32, 100 filters with size 3 in the convolutional layer, and 200 as memory dimension for LSTM. For regular users, the hyperparameters are the same, except in the LSTM layer, where a memory dimension of 100 was used. For experts, CNN-GRU uses 100 filters with size 5 as filter length and 3 as pool size for both CNNs. In the GRU, we used dimensionality of 150 and batch size of 16. For regular users, the differences are that we used a dimensionality of 100 in the GRU layer, size 3 as filter length and 2 as pool size for both CNNs. For both C-LSTM and CNN-GRU the differences in the hyperparameters are explained by the fact that our expert reviews are significantly shorter than the ones wrote by regular users. After selecting the best hyperparameters, we trained two models for each architecture, one for experts and other for regular users. Also, each result reported in the paper is the average of five runs, where for each run the model is trained from start using the whole training dataset. With that, we can measure their parameter sensitivity and calculate confidence intervals for the results. In addition, C-LSTM and CNN-GRU took approximately half a day to train and BERT one day. Finally, we noted that the performance of all three models were not significantly affected by the hyperparameter configurations we tested. 